\title{
Robust Rhythmogenesis via Spike Timing Dependent Plasticity
}

\author{
Gabi Socolovsky ${ }^{1,2, *}$ and Maoz Shamir ${ }^{1,2,3}$ \\ ${ }^{1}$ Department of Physics, Faculty of Natural Sciences, \\ ${ }^{2}$ Zlotowski Center for Neuroscience, \\ ${ }^{3}$ Department of Physiology and Cell Biology, Faculty of Health Sciences, \\ Ben-Gurion University of the Negev, Be'er-Sheva, Israel
}

(Dated: March 31, 2021)

\begin{abstract}
Rhythmic activity has been observed in numerous animal species ranging from insects to humans, and in relation to a wide range of cognitive tasks. Various experimental and theoretical studies have investigated rhythmic activity. The theoretical efforts have mainly been focused on the neuronal dynamics, under the assumption that network connectivity satisfies certain fine-tuning conditions required to generate oscillations. However, it remains unclear how this fine tuning is achieved.

Here we investigated the hypothesis that spike timing dependent plasticity (STDP) can provide the underlying mechanism for tuning synaptic connectivity to generate rhythmic activity. We addressed this question in a modeling study. We examined STDP dynamics in the framework of a network of excitatory and inhibitory neuronal populations that has been suggested to underlie the generation of oscillations in the gamma range. Mean field Fokker Planck equations for the synaptic weights dynamics are derived in the limit of slow learning. We drew on this approximation to determine which types of STDP rules drive the system to exhibit rhythmic activity, and demonstrate how the parameters that characterize the plasticity rule govern the rhythmic activity. Finally, we propose a novel mechanism that can ensure the robustness of self-developing processes, in general and for rhythmogenesis in particular.
\end{abstract}

\section{INTRODUCTION}

Rhythmic activity in the brain has been observed for more than a century $[1,2]$. Oscillations in different frequency bands have been associated with different cognitive tasks and mental states [2-6]. Specifically, rhythmic activity in the Gamma band has been described in association with sensory stimulation [7], attentional selection $[8,9]$, working memory [10] and other measures [11]. Deviation from normal rhythmic activity has been associated with pathology [12-15].

Considerable theoretical efforts have been devoted to unraveling the neural mechanism responsible for generating rhythmic activity in general [2] and in the Gamma band in particular [16-21]. One possible mechanism is based on delayed inhibitory feedback [21-24]. The basic architecture of this mechanism is composed of one excitatory and one inhibitory neuronal populations, with reciprocal connections (Fig. 1a). A target rhythm is obtained by tuning the strengths of the excitatory and inhibitory interactions (Fig. 1b-1c). However, it is unclear which mechanism results in the required fine-tuning $[23,25]$.

We hypothesized that activity dependent synaptic plasticity can provide the mechanism for tuning the interaction strengths in order to stabilize a specific rhythmic activity in the gamma band.

Here we focused on spike timing dependent plasticity (STDP) as the rhythmogenic process $[23,25]$. Below, we briefly describe STDP and derive the dynamics of the synaptic weights in the limit of slow learning.

* gabisoco@post.bgu.ac.il
Since the cross-correlation of neural activity is central to STDP dynamics, we next define the network dynamics and analyze its phase diagram and the dependence of the correlations on the synaptic weights. Using the separation of timescales in the limit of slow learning we analyze STDP dynamics and investigate under what conditions STDP can stabilize a specific rhythmic activity and how the characteristics of the STDP rule govern the resultant rhythmic activity. Finally, we summarize our results, discuss possible extensions and limitations and propose a general principle for robust rhythmogenesis.

\section{STDP DYNAMICS}

The basic coin of information transfer in the central nervous system is the spike: a short electrical pulse that propagates along the axon (output branch) of the transmitting neuron to synaptic terminals that relay the information to the dendrites (input branch) of the receiving neurons downstream. While spikes are stereotypical, the relayed signal depends on the synaptic weight, which can be thought of as interaction strength. Learning is the process that modifies synaptic weights (the dynamics of the interaction strengths themselves), and typically occurs on a slower timescale than the timescale of the neuronal responses.

STDP is an empirically observed microscopic learning rule in which the modification of the synaptic weight depends on the temporal relation between the spike times of the pre (transmitting) and post (receiving) synaptic neurons [26-30]. Following [31] the change, $\Delta J_{i j}$, in the synaptic weight $J_{i j}$ from the pre-synaptic neuron $j$ to the post-synaptic neuron $i$ is expressed as the sum of two pro- 


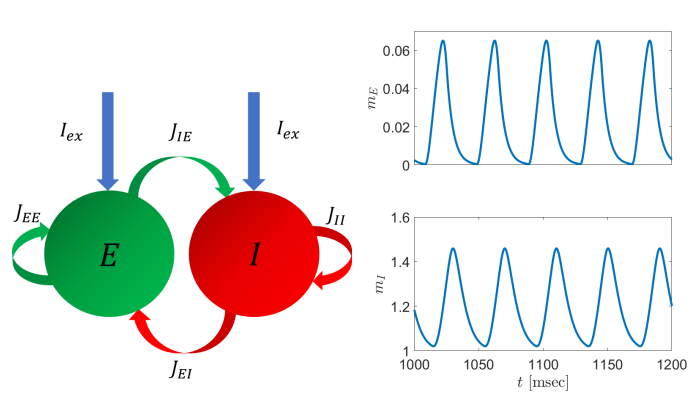

(a)

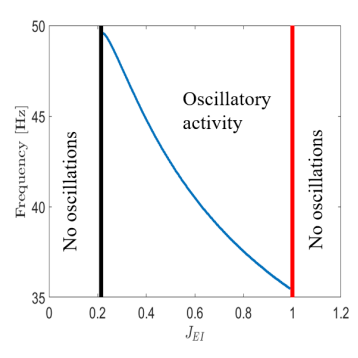

(c)

(d)

FIG. 1: The excitatory-inhibitory network. (a) Model architecture. The neuronal network here is composed of an excitatory (E) and an inhibitory (I) populations with inter$\left(J_{I E}\right.$ and $\left.J_{E I}\right)$ and intra- $\left(J_{I I}\right.$ and $J_{E E}$, that will be taken to zero hereafter) connections. The interaction is not symmetric and is delayed. (b) The oscillatory dynamics of the mean excitatory end inhibitory population firing rates $m_{E}$ and $m_{I}$, respectively, at a frequency of $24.9 \mathrm{~Hz}$. Here we used $J_{E E}=J_{I I}=0, J_{I E}=8.91, J_{E I}=0.9, \tau_{m}=d=5 \mathrm{~ms}$. (c) Rhythmic activity. The oscillation frequency is depicted as a function of the strength of the inhibitory to excitatory connection, $J_{E I}$. The following parameters were implemented here: $J_{E E}=J_{I I}=0, J_{I E}=8.91, \tau_{m}=10 \mathrm{~ms}$, $d=1 \mathrm{~ms}$. (d) Phase diagram of the delayed rate model. Strong inhibition, $J_{I}>1$, leads the system to a purely inhibitory fixed point, in which $m_{E}$ is fully suppressed, and $m_{I}=1$. For weak to moderate inhibition, $J_{I} \in(0,1)$, and below the black line, the system converges to a fixed point, where both populations are active. For $\bar{J}>\bar{J}_{d}$ (above the black line) the model exhibits rhythmic activity. For a given time delay, the frequency is governed by $\bar{J}$. When $\bar{J}$ is increased, higher frequencies are observed. Here we used $\tau_{m}=d=1$.

cesses: potentiation (i.e., increasing the synaptic weight) and depression (i.e., decreasing the synaptic weight):

$$
\Delta J_{i j}=\lambda\left[K_{+}(\Delta t)-\alpha K_{-}(\Delta t)\right],
$$

where $\Delta t=t_{i}-t_{j}$ is the pre and post spike time difference. Functions $K_{ \pm}(t) \geq 0$ describe the temporal structure of the potentiation $(+)$ and depression $(-)$ of the STDP rule. Parameter $\alpha$ denotes the relative strength of the depression and $\lambda$ is the learning rate. We assume that learning occurs on a slower timescale than the characteristic timescales that describe neuronal activity (for given fixed synaptic weights).

A wide range of temporal structures of STDP rules has been reported [32-39]. Here we focus on two families of rules. One is composed of temporally symmetric rules and the other is made up of temporally asymmetric rules.

For the temporally symmetric family we apply a difference of Gaussian STDP rule; namely:

$$
K_{ \pm}(t)=\frac{1}{\sqrt{2 \pi} \tau_{ \pm}} e^{-\left(t / \tau_{ \pm}\right)^{2} / 2}
$$

where $\tau_{ \pm}$denotes the characteristic time scales of the potentiation $(+)$ and depression $(-)$. Consistent with the popular description of the famous Hebb rule that 'neurons that fire together wire together' [40], we refer to the case of $\tau_{+}<\tau_{-}$as Hebbian, and $\tau_{+}<\tau_{-}$antiHebbian.

For the temporally a-symmetric STDP rules we take:

$$
K_{ \pm}(t)=\frac{1}{\tau_{ \pm}} e^{\mp H t / \tau_{ \pm}} \Theta( \pm H t)
$$

where $\Theta(x)$ is the Heaviside step function, $\tau_{ \pm}$are the characteristic time scales of the potentiation $(+)$ and depression (-), and $H= \pm 1$ dictates the Hebbianity of the STDP rule. The rule will be termed Hebbian for $H=1$, when potentiation occurs in the causal branch, $t_{\text {post }}>t_{\text {pre }}$, and anti-Hebbian for $H=-1$.

Different types of synapses have been reported to exhibit different types of STDP rules. Consequently, there is no a-priori reason to assume that excitatory and inhibitory synapses share the exact same learning rule. In particular, the characteristic time constants $\tau_{E, \pm}$ for excitatory synapses and $\tau_{I, \pm}$ for inhibitory synapses may differ.

Changes to synaptic weights due to the plasticity rule of Eq. (1) at short time intervals occur as a result of either a pre or post-synaptic spike during this interval. Thus,

$$
\begin{aligned}
\dot{J}_{i, j}(t) & =\lambda \rho_{i}(t) \int_{0}^{\infty} \rho_{j}\left(t-t^{\prime}\right)\left[K_{+}\left(t^{\prime}\right)-\alpha K_{-}\left(t^{\prime}\right)\right] d t^{\prime} \quad(4) \\
& +\lambda \rho_{j}(t) \int_{0}^{\infty} \rho_{i}\left(t-t^{\prime}\right)\left[K_{+}\left(-t^{\prime}\right)-\alpha K_{-}\left(-t^{\prime}\right)\right] d t^{\prime},
\end{aligned}
$$

where $\rho_{\mathrm{post} / \mathrm{pre}}(t)=\sum_{l} \delta\left(t-t_{l}^{\mathrm{post} / \mathrm{pre}}\right)$ is the spike train of the post/pre neuron written as the sum of the delta function at the neuron's spike times $\left\{t_{l}^{\text {post } / \text { pre }}\right\}_{l}$. In the limit of slow learning, $\lambda \rightarrow 0$, the right hand side of Eq. (4) can be replaced by its temporal mean (see [31] for complete derivations). This approximation, has been termed the mean field Fokker-Planck approximation [27]. As fluctuations vanish in this limit and deterministic dynamics are retained for the mean synaptic weights, we get

$$
\dot{J}_{i j}(t)=\lambda \int_{-\infty}^{\infty} \Gamma_{i j}\left(-t^{\prime}\right)\left[K_{+}\left(t^{\prime}\right)-\alpha K_{-}\left(t^{\prime}\right)\right] d t^{\prime},
$$


where $\Gamma_{i j}(t)$ is the cross correlation of neurons $i$ and $j$ :

$$
\Gamma_{i j}(t)=\left\langle\rho_{i}\left(t^{\prime}\right) \rho_{j}\left(t^{\prime}+t\right)\right\rangle \text {. }
$$

The angular brackets $\langle\cdots\rangle$ denote ensemble averaging over the neurnal noise and temporal averaging over one period in the case of rhythmic activity (see Soloduchin [23] \& Shamir and Luz \& Shamir [31] for more details). Note that the dependence of the r.h.s. of Eq. (5) on time, $t$, occurs through the dependence of the cross-correlations on the synaptic weights at time $t$. Thus, the key to analyzing STDP dynamics is the ability to compute the cross-correlations of the neural activities and grasp their dependence on the synaptic weights. To this end we examined rhythmogenesis in the gamma band using the framework of a reduced rate model with delay, proposed by Roxin and colleagues (Fig. 1a). A complete analysis of the model appears in $[21,22,24]$. Below we briefly describe the phase diagram of the system and derive the cross-correlations.

\section{THE DELAYED EXCITATORY INHIBITORY NETWORK}

The firing of different neurons is assumed to follow independent inhomogeneous Poisson process statistics with instantaneous firing rates that adhere to the reduced model in Roxin et al. [21]. In their work, Roxin and colleagues considered a full model that included interpopulation as well as intra-population interactions (i.e., excitatory-excitatory and inhibitory-inhibitory). Here, for simplicity we restrict the analysis to the minimal model that can reproduce oscillations in the gamma band. To do so, we model the rate dynamics of the gamma generating network by:

$$
\begin{aligned}
\tau_{m} \dot{m}_{E}(t) & =-m_{E}(t)+\left[I-J_{I} m_{I}(t-d)\right]_{+} \\
\tau_{m} \dot{m}_{I}(t) & =-m_{I}(t)+\left[I+J_{E} m_{E}(t-d)\right]_{+},
\end{aligned}
$$

where $m_{E / I}(t)$ is the mean firing rate of the excitatory (E) and inhibitory (I) population at time $t . \tau_{m}$ is the neuronal time constant. Unless stated otherwise, we take $\tau_{m}=1$, which is equivalent to measuring time in units of the neuronal time constant. Parameter $d$ denotes the delay, and $I$ is the external input to the system. In our analysis we took $I=1 . J_{E}$ and $J_{I}$ are the effective interaction strengths between the two populations. $J_{E}\left(J_{I}\right)$ can be thought of as a global order parameter reflecting the mean synaptic weight from the excitatory (inhibitory) pre-synaptic population to the inhibitory (excitatory) post-synaptic population.

For strong inhibition, $J_{I}>1$, the system converges to a fixed point in which the excitatory population is fully suppressed by the inhibitory population, $\vec{m}^{*}=\left(\begin{array}{l}0 \\ 1\end{array}\right)$. For weak to moderate levels of inhibition, $J_{I} \in(0,1)$, the system has a fixed point in which both populations are active, $\vec{m}^{*} \equiv\left(\begin{array}{c}m_{E}^{*} \\ m_{I}^{*}\end{array}\right)=\frac{1}{1+\bar{J}^{2}}\left(\begin{array}{c}1-J_{I} \\ 1+J_{E}\end{array}\right)$, with $\bar{J} \equiv \sqrt{J_{E} J_{I}}$.
However, this fixed point is not stable for $\bar{J}>\bar{J}_{d}$, where $\bar{J}_{d}^{2}=1+\omega_{d}^{2}, \omega_{d}=\cot \left(\omega_{d} d\right)$ and $\omega_{d} \in[0, \pi / 2 d]$ (see Roxin et al. [21]). In this region $\left(\bar{J}>\bar{J}_{d}\right.$ and $\left.J_{I}<1\right)$ the system converges to a limit cycle solution, Fig. 1d.

By rescaling the firing rates, the two dimensional first order delayed dynamics, Eq. (7)-(8), can be reduced to a one dimensional delayed dynamic equation:

$$
\ddot{x}(t)+2 \dot{x}(t)+x(t)-\left[1-\bar{J}^{2} x(t-2 d)\right]_{+}=0,
$$

with

$$
\begin{aligned}
m_{I}(t) & =1+\left(J_{E}-\bar{J}^{2}\right) x(t) \\
m_{E}(t-d) & =\frac{J_{E}-\bar{J}^{2}}{J_{E}}(x(t)+\dot{x}(t)) .
\end{aligned}
$$

Equation (9) highlights the fact that the temporal structure of the limit cycle solution depends on the synaptic weights, $J_{E}$ and $J_{I}$, only via $\bar{J}$. In particular, the period of oscillations is solely a function of $\bar{J}$ and $d$. As shown in Fig. 2a the period is a monotonically increasing function of both $\bar{J}$ and $d$.

In our model the cross-correlations are given by temporal averaging of the mean firing rates, $\Gamma_{I E}(\Delta) \equiv$ $\left\langle m_{I}(t) m_{E}(t+\Delta)\right\rangle$. In the fixed point region of the phase diagram, the cross-correlation is simply given by the product of the mean rates, $\Gamma_{I E}(\Delta)=$ $\left(1-J_{I}\right)\left(1+J_{E}\right) /\left(1+\bar{J}^{2}\right)^{2}$.

In the region of the phase diagram where the system converges to a limit cycle solution, Eqs. (10) \& (11) provide the scaling of the cross-correlations; namely,

$$
\Gamma_{I E}(\Delta)=a \bar{x}+b\left(\Gamma_{x}(\Delta+d)+\frac{d}{d \Delta} \Gamma_{x}(\Delta+d)\right)
$$

where $a \equiv\left(J_{E}-\bar{J}^{2}\right) / J_{E}, b \equiv\left(J_{E}-\bar{J}^{2}\right)^{2} / J_{E}, \bar{x} \equiv$ $\langle x(t)\rangle$, and $\Gamma_{x}(s) \equiv\langle x(t) x(t+s)\rangle$. Note that $\bar{x}$ and $\Gamma_{x}$ depend solely on the delay, $d$, and $\bar{J}$. Numerical investigation reveals that the auto-correlation of $x(t)$ is well approximated by a cosine function,

$$
\Gamma_{x}(s) \approx \bar{\Gamma}_{x}+\tilde{\Gamma}_{x} \cos (\omega s)
$$

where we used

$$
\begin{aligned}
& \bar{\Gamma}_{x}=\int_{0}^{T} \Gamma_{x}(s) d s / T \\
& \tilde{\Gamma}_{x}=2 \int_{0}^{T} \Gamma_{x}(s) \cos (2 \pi s / T) d s / T,
\end{aligned}
$$

with $T$ denoting the period of the limit cycle, as can be seen from the value of $R^{2}$, Fig. $2 \mathrm{~b}$. The goodness of fit of the cosine approximation decreases when $\bar{J}$ or $d$ are increased. Nevertheless, for a wide range of parameters relevant to the generation of gamma oscillations $R^{2}$ is extremely high $\left(R^{2}>0.98\right.$ throughout Fig. $\left.2 \mathrm{~b}\right)$. Both $\bar{x}$ and $\bar{\Gamma}_{x}$ monotonically decrease as $\bar{J}$ increases, Fig. 2 c-2d. In addition, they transition the bifurcation line continuously. On the other hand, $\tilde{\Gamma}_{x}$ does not transition in a continuous manner: it is zero in the fixed point region 


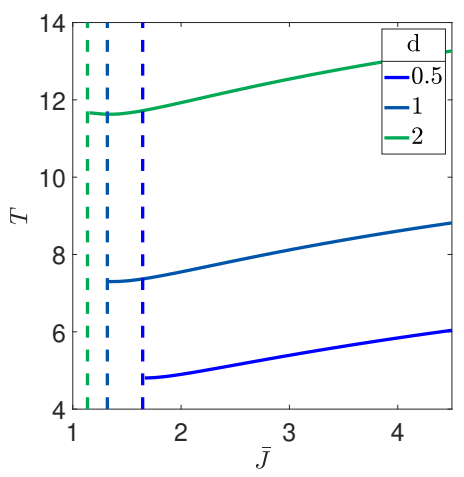

(a)

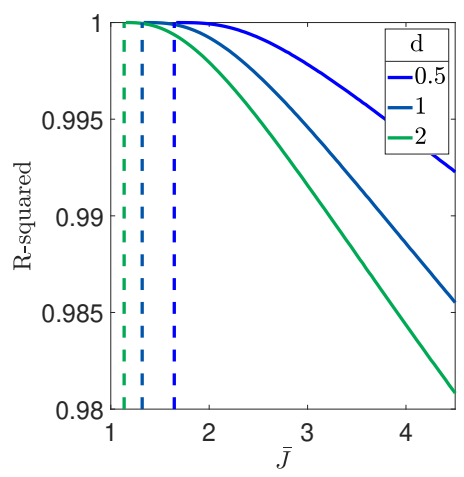

(b)

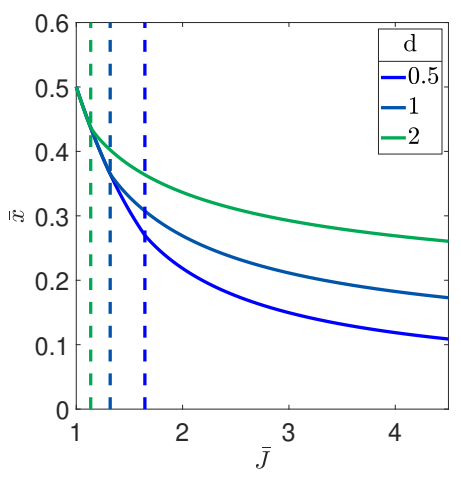

(c)

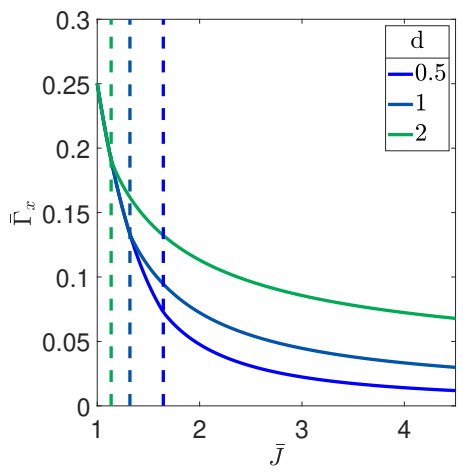

(d)

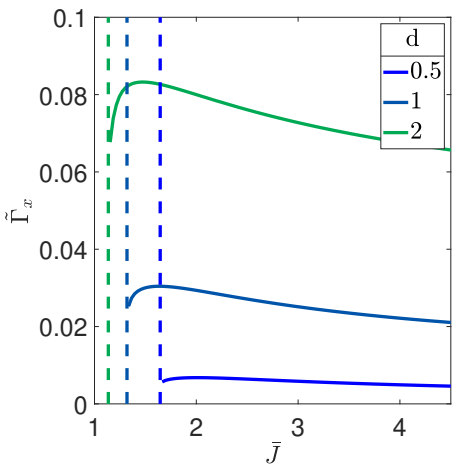

(e)

FIG. 2: Dynamics of the one dimensional variable $x$, Eq. (9). Different features characterizing the dynamics of $x$ are shown as a function of $\bar{J}$, for different values of the delay, $d$, differentiated by color. The dashed lines indicate the values of $\bar{J}_{d}$, for different delays, shown by color. The parameters plotted are: (a) The period of the oscillations. (b) The goodness of fit of the cosine approximation, Eq. (13), of $\Gamma_{x} . R^{2}$. (c) The temporal average of $x, \bar{x}$. (d) The zeroth order Fourier component of the auto-correlation of $x, \bar{\Gamma}_{x}$. (e) The first order Fourier component of the auto-correlation of $x, \tilde{\Gamma}_{x}$.

and jumps to a positive value in the rhythmic region, Fig. 2e.

Using the cosine approximation for the correlations, Eq. (13) and the scaling Eqs. (10) \& (11) yields the semiempirical excitatory-inhibitory cross-correlations function:

$$
\Gamma_{I E}(\Delta) \approx \bar{\Gamma}+\tilde{\Gamma} \cos (\omega \Delta+\tilde{\varphi})
$$

with $\bar{\Gamma}=a \bar{x}+b \bar{\Gamma}_{x}, \tilde{\Gamma}=b \tilde{\Gamma}_{x}\left(1+\omega^{2}\right)^{1 / 2}, \tilde{\varphi}=\omega\left(d+\varphi_{\omega}\right)$ and $\omega \varphi_{\omega}=\arcsin \left(\omega / \sqrt{1+\omega^{2}}\right)$. Figure 3 shows the values of $\tilde{\varphi}$ on the phase diagram. The phase, $\tilde{\varphi}(\bar{J}, d)$ is $\pi / 2$ on the bifurcation line and weakly decreases as $\bar{J}$ is further increased. Note that $\Gamma_{E I}(\Delta)=\Gamma_{I E}(-\Delta)$.

\section{STDP INDUCED FLOW ON THE PHASE DIAGRAM}

Utilizing the semi-empirical cross-correlations, Eq. (16), yields the following dynamics for the synaptic weights:

$$
\dot{J}_{\sigma}=\lambda\left(\bar{\Gamma} \bar{K}+\tilde{\Gamma} \tilde{K}_{\sigma}\right)
$$

where

$$
\bar{K}=\int_{-\infty}^{\infty} K(\Delta) d \Delta
$$

$$
\tilde{K}_{\sigma} \equiv \int_{-\infty}^{\infty} K(\Delta) \cos \left(\omega \Delta_{\sigma}+\tilde{\varphi}\right) d \Delta,
$$

where $\sigma=E, I$, we used the notation $K(\Delta)=K_{+}(\Delta)-$ $\alpha K_{-}(\Delta)$, and $\Delta_{E}=-\Delta$ whereas $\Delta_{I}=\Delta$. 


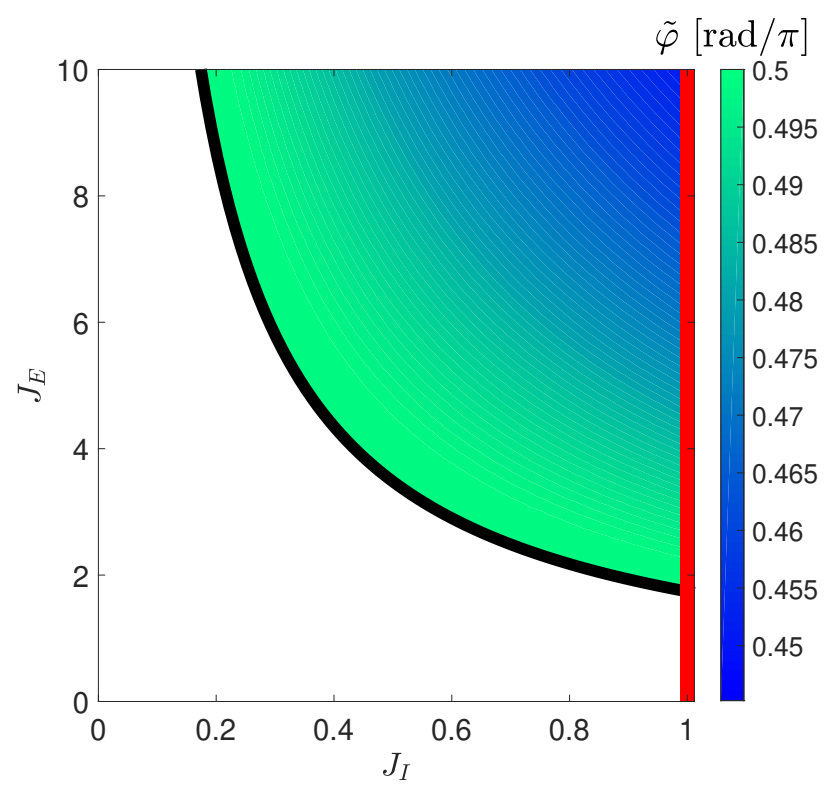

FIG. 3: The values of $\tilde{\varphi}$. The parameter, $\tilde{\varphi} / \pi$ is shown by color as a function of $J_{E}$ and $J_{I}$ on the phase diagram. Here $d=1$ was used.

The STDP dynamics, Eq. (17), induces a flow in the phase plane of the synaptic weights $\left[J_{I}, J_{E}\right]$, which is also the phase diagram of the neural responses. The right hand side of Eq. (17) depends on the synaptic weights through the Fourier transforms of the cross-correlations $\bar{\Gamma}$ and $\tilde{\Gamma}$. Using the separation of timescales between the fast neuronal responses and the slow learning rate, in the limit of slow learning $\lambda \rightarrow 0$, one can compute $\bar{\Gamma}$ and $\tilde{\Gamma}$ from the neuronal dynamics for fixed synaptic weights.

Thus, STDP induces a flow on the phase diagram of the system. Rhythmogenesis is obtained when this flow guides the system and stabilizes it at a fixed point on the phase diagram that is characterized by the desired rhythm.

In the region of the phase diagram in which the mean neuronal firings relax to a fixed point, $\tilde{\Gamma}=0$. Due to our choice of normalization, $\bar{K}_{ \pm}=1$, in this region $\operatorname{sign}\left(\dot{J}_{\sigma}\right)=\operatorname{sign}\left(1-\alpha_{\sigma}\right), \sigma \in\{E, I\}$. Consequently, the STDP dynamics will induce a flow from the fixed point region towards the rhythmic region if and only if the potentiation is strong relative to the depression for both types of synapses, $\alpha_{E}, \alpha_{I}<1$ (except for a small region of the phase diagram with high inhibition and low excitation, which also depends on the learning rates, $\lambda_{E}$ and $\lambda_{I}$, of the different synapses). This result holds true for any STDP rule. In contrast, the STDP dynamics in the rhythmic region of the phase diagram depend on the temporal structure of the learning rule.

The difference of Gaussians learning rule, Eq. (2), yields

$$
\tilde{K}_{\sigma}=\cos \tilde{\varphi}\left(e^{-\frac{\left(\omega \tau_{\sigma,+}\right)^{2}}{2}}-\alpha e^{-\frac{\left(\omega \tau_{\sigma,-}\right)^{2}}{2}}\right),
$$

with $\sigma \in\{E, I\}$. Consequently, the dynamical equations of $J_{E}$ and $J_{I}$ will be identical if the characteristic time scales of potentiation and depression are the same; namely, if $\tau_{E,+}=\tau_{I,+}$ and $\tau_{E,-}=\tau_{I,-}$. Note that on the r.h.s. of Eq. (20), the term $\cos \tilde{\varphi}$ ensures that $\tilde{K}_{\sigma}$ is zero on the bifurcation (see Fig. 3). Figures 4a and $4 \mathrm{~b}$ depict the nullclines of $J_{E}$ and $J_{I}$, respectively, for different values of the relative strength of depression, $\alpha$ (in 4a), and the characterstic time of depression, $\tau_{-}$(in 4b), differentiated by color. We show that for $\alpha<1$ $(\alpha>1)$ and $\tau_{+}>\tau_{-}\left(\tau_{+}<\tau_{-}\right)$the nullcline of $J_{E}$ and the left branch of the nullcline of $J_{I}$ are stable (unstable). A fixed point of the STDP dynamics is obtained by the intersection of $J_{E}$ and $J_{I}$ nullclines. For the difference of Gaussians rule, a stable fixed point that exhibits rhythmic activity in the gamma band can thus be obtained. However, this requires a delicate adjustment of the parameters characterizing the STDP learning rules.

For the temporally asymmetric Hebbian exponential rule, Eq. (3) with $H=1$, we obtain

$$
\begin{aligned}
\tilde{K}_{\sigma}= & \cos \left(\theta_{\sigma,+}\right) \cos \left(\theta_{\sigma,+}-\tilde{\varphi}_{\sigma}\right) \\
& -\alpha \cos \left(\theta_{\sigma,-}\right) \cos \left(\theta_{\sigma,-}+\tilde{\varphi}_{\sigma}\right)
\end{aligned}
$$

where $\cos \left(\theta_{\sigma, \pm}\right)=\left(1+\left(\omega \tau_{\sigma, \pm}\right)^{2}\right)^{-1 / 2}, \tilde{\varphi}_{E}=\tilde{\varphi}$ and $\tilde{\varphi}_{I}=-\tilde{\varphi}$. Now, due to $\theta_{\sigma, \pm}, \tilde{K}_{\sigma}$ transitions discontinuously across the bifurcation line, thus inducing discontinuity in $\dot{J}_{E}$ and $\dot{J}_{I}$ along the transition from fixed point to the rhythmic region. Figures $4 \mathrm{c}$ and $4 \mathrm{~d}$ depict the nullclines of $J_{I}$, for the temporally asymmetric Hebbian learning rule for different values of the relative strength of depression, $\alpha$ (in 4c), and the characteristic time of depression, $\tau_{-}$(in $4 \mathrm{~d}$ ), differentiated by color. The left branch of the nullclines of $J_{I}$ is stable (unstable) for $\alpha<1(\alpha>1)$ and $\tau_{+}<\tau_{-}\left(\tau_{+}>\tau_{-}\right)$. Interestingly, a considerable part of the $J_{I}$ nullcline is on the bifurcation line.

For the temporally asymmetric Hebbian learning rule, the dynamics of $J_{E}$ do not have a nullcline. As a result, a fixed point does not exist, and the temporally asymmetric Hebbian STDP rule cannot stabilize rhythmic activity in the gamma range.

However, as $\Gamma_{E I}(\Delta)=\Gamma_{I E}(-\Delta)$ the temporally asymmetric anti-Hebbian exponential rule (Eq. (3) with $H=-1$ ) for excitatory synapses, $J_{E}$, in our model, defines the exact same dynamics as that of an inhibitory synapses, $J_{I}$, with a Hebbian rule (Eq. (3) with $H=+1$ ). Therefore, an asymmetric Hebbian learning rule for $J_{I}$ and an asymmetric anti-Hebbian learning rule for $J_{E}$ yield the same nullclines (see Fig. 4c-4d). Moreover, because a considerable part of the nullcline is on the bifurcation line, no fine tuning of the parameters is required to obtain a fixed point of the STDP dynamics that will generate rhythmic activity at $\omega_{d}$. Thus, the STDP dynamics have a line attractor on (part of) the bifurcation line. Furthermore, when the left branches of the nullclines of $J_{E}$ and $J_{I}$ are stable $\left(\alpha<1, \tau_{E,-}>\tau_{E,+}\right.$ and $\left.\tau_{I,-}>\tau_{I,+}\right)$ and the nullcline of $J_{I}$ departs from the 


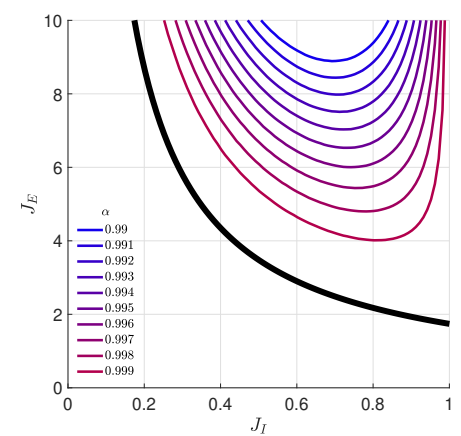

(a)

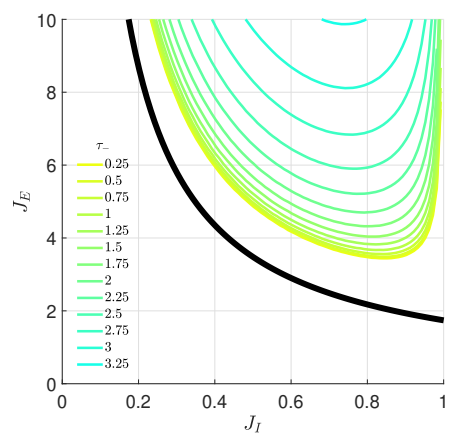

(b)

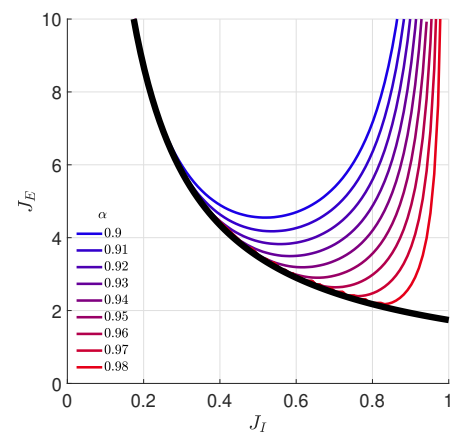

(c)

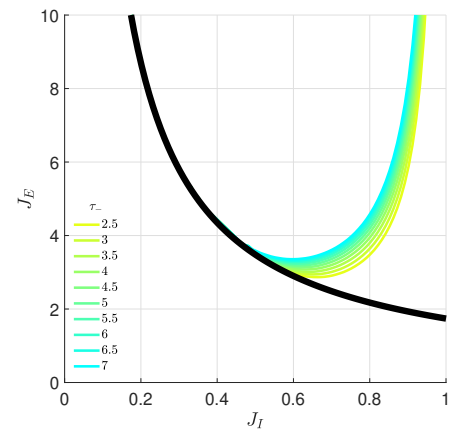

(d)

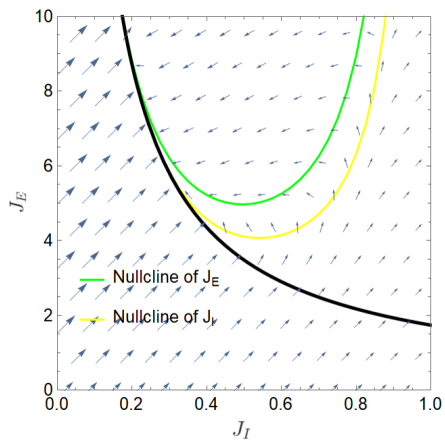

(e)

FIG. 4: Nullclines of the STDP dynamics. (a) and (b) The nullclines of $J_{E}$ and $J_{I}$ for the difference of Gaussians learning rule (in this case the nullclines are identical for the same choice of parameters $\alpha, \tau_{ \pm}$) The nullclines are shown for different values of of $\alpha=0.99,0.991, \ldots 0.999$, differentiated by color, with $\tau_{+}=2$ and $\tau_{-}=1$, in (a). The nullclines are shown for different values of $\tau_{-}=0.25,0.5, \ldots 3.25$, by colors, with $\tau_{+}=5$ and $\alpha=0.999$, in (b). (c) and (d) The nullclines of $J_{I}\left(J_{E}\right)$ for the temporally asymmetric Hebbian (anti-Hebbian) STDP rule. The nullclines are shown for different values of $\alpha=0.9,0.91, \ldots 0.99$, with $\tau_{+}=2$ and $\tau_{-}=5$, in (c). The nullclines are shown for different values of $\tau_{-}=2.5,3, \ldots 7$, with $\tau_{+}=2$ and $\alpha=0.94$, in (d). The nullclines were computed using the cosine approximation for the neuronal cross-correlations. (e) The vector flow in the case of a temporally asymmetric Hebbian learning rule for $J_{I}$ and a temporally asymmetric anti-Hebbian learning rule for $J_{E}$. The nullclines are shown for $\alpha=0.88, \tau_{I,+}=\tau_{E,+}=1, \tau_{I,-}=2$ and $\tau_{E,-}=5$. The learning rates are $\lambda_{I}=1$ and $\lambda_{E}=10$. In every subfigure $d=1$ was used.

bifurcation line below the nullcline of $J_{E}$, the attractor line is stable against perturbations in every direction (See Fig. 4e).

\section{DISCUSSION}

Previous studies have investigated the effects of rhythmic activity on STDP [20, 41-50]. However, in these studies, rhythmic activity was hard-wired in the system and the issue of rhythmogenesis was not addressed.

Rhythmogenesis can be thought of as self organizing temporal activity; i.e., the ability of a non-rhythmic system to spontaneously develop rhythmic activity. In our approach, the process of rhythmogenesis was mapped to a flow on the phase diagram. This mapping relies on the separation of timescales.
Previously, Soloduchin \& Shamir investigated rhythmogenesis using the framework of two neuronal populations with reciprocal inhibition and short term adaptation in the form of firing rate adaptation $[23,25]$. The network motif of reciprocal inhibition has been widely reported in the central nervous system [51-53]. However, it is mainly associated with winner-take-all like competition [54-60] rather than generating rhythmic activity (but see [61, 62] in the spinal cord). Here, rhythmogenesis was studied in the framework of a network that is considered a valid hypothesis for generating gamma rhythm in the brain $[21,22,24]$.

In Soloduchin \& Shamir [23], rhythmogenesis was obtained as a specific stable fixed point on the phase diagram of the system, in which due to the temporal characteristics of the STDP rule, the dynamics of the synaptic weights vanish at a specific frequency. This scenario is similar to the case of temporally symmetric STDP (Fig. 
4a-4b). However, scientifically, this scenario is somewhat disappointing, since we have traded the problem of finetuning of the synaptic weights for the problem of finetuning of characteristics of the STDP rule [25].

The temporally asymmetric STDP rule provides a possible solution to the fine-tuning problem of rhythmogenesis, which we term critical rhythmogenesis (Fig. 4c-4d). Rhythmogenesis in the temporally asymmetric STDP rule is not obtained as a fixed point of the STDP dynamics. Rather, in this case, rhythmogenesis utilizes the discontinuity of $\tilde{\Gamma}$ across the bifurcation line. For a wide range of parameters the flow induced by the STDP is directed from the fixed point region towards the rhythmic region and from the rhythmic region to the fixed point region, and the system will settle on the bifurcation line itself. Consequently, the resultant rhythmic activity will be dictated by bifurcation (e.g., the firing rates will oscillate at $\omega_{d}$ ), which is independent of the synaptic plasticity thus accounting for the robustness to the parameters that characterize the STDP.

Recently, Pernelle and colleagues studied the possible contribution of gap junction plasticity to rhythmic activity [63]. They postulated a plasticity rule for gap junctions that tuned the system to operate on the boundary of asynchronous regular firing activity, on one hand, and rhythmic activity of synchronous bursts, on the other. In the context of our work this can be viewed as an example of critical rhythmogenesis, which explains the robustness of their putative mechanism to variations in the plasticity rule.

We suggest that the scenario of critical rhythmogenesis may provide a general principle for robustness in biological systems. Assume a certain biological system, which is characterized by set parameters: $\left\{x_{1}, x_{2} \ldots x_{n}\right\}$ (i.e., $\vec{x}$ is a point in the phase diagram of the system), is required to maintain a certain living condition, $f\left(\left\{x_{1}, x_{2} \ldots x_{n}\right\}\right)=0$. This condition is met by a homeostatic process. The homeostatic process defines the dynamics on $\left\{x_{1}, x_{2} \ldots x_{n}\right\}$, which are characterized by another set of parameters, $\left\{\alpha_{1}, \alpha_{2} \ldots \alpha_{m}\right\}$, namely: $\dot{\vec{x}}=\mathcal{F}(\vec{x}, \vec{\alpha})$. A viable homeostatic process is a choice of parameters, $\vec{\alpha}^{*}$, such that the homeostatic dynamics will lead the system to a set of parameters, $x_{\infty}$, that satisfy the living condition, $f\left(\vec{x}_{\infty}\right)=0$.

How can this be achieved? One possibility is that $x_{\infty}$ is a stable fixed point of the homeostatic dynamics, in which $\mathcal{F}\left(\vec{x}_{\infty}, \vec{\alpha}^{*}\right)=0$. This solution requires fine-tuning of the parameters that define the homeostatic process, $\vec{\alpha}$. In this case, fluctuations in $\vec{\alpha}$ will generate fluctuations in $\vec{x}$ away from $x_{\infty}$.

An alternative scenario is that the homeostatic dynamics utilize some discontinuity in the phase diagram. In this scenario the dynamics do not necessarily vanish on $x_{\infty}, \mathcal{F}\left(\vec{x}_{\infty}, \vec{\alpha}^{*}\right) \neq 0$. Rather, due to the discontinuity there exists a wide range of parameters, $\left\{\alpha_{1}, \alpha_{2} \ldots \alpha_{m}\right\}$, such that the dynamics draw the system towards the discontinuity from both sides, as is the case for critical rhythmogenesis. Consequently, this scenario can stabilize the system in a critical condition on the boundary of two phases.

The idea that the central nervous system may operate in (or near) a critical condition has been suggested in the past, and may have computational advantages [6466]. However, this latter scenario also has shortcomings. The most obvious is that it can only be used to ensure and stabilize critical behavior. In addition, it cannot be used when one of the phases near the critical line is lethal.

An advantage of critical rhythmogenesis is that it allows for rapid switching between rhythmic and nonrhythmic phases, for example by neuromodulators, since the system is on the boundaries of these phases. This raises the question of the likelihood of critical rhythmogenesis: what is the probability that a biological system will 'choose' the exact rhythmic activity that also characterizes the bifurcation? One possible explanation is that the opposite took place. In other words, biological systems have evolved to operate at the critical conditions chosen by the critical rhythmogenesis mechanism. Thus, in our example, the characteristic delays, $d$, do not miraculously fit the desired rhythm. Rather, due to the specific values of $d$, the critical rhythmogenesis tunes the system to oscillate at $\omega_{d}$ which is why the biological system 'uses' this specific frequency band.

In our work we made several simplifying assumption to facilitate the analysis. We studied the dynamics of the effective couplings between excitatory and inhibitory populations and did not incorporate the STDP dynamics of individual synapses. We investigated a model with only inter-connections, hence ignoring the effective couplings that represent the intra-population synapses. We estimated neuronal correlations using a simplified rate model, and did not study the effects of spiking neurons. These issues are beyond the scope of the current study and will be addressed elsewhere. Nevertheless, this work lays the foundation for studying a novel mechanism for robust homeostatic plasticity, in general, and rhythmogenesis in particular.

\section{ACKNOWLEDGMENTS}

This work was supported by the Israel Science Foundation, grant number 300/16.
[1] R Jung and W Berger. Fiftieth anniversary of hans berger's publication of the electroencephalogram. his first records in 1924-1931 (author's transl). Archiv fur Psychiatrie und Nervenkrankheiten, 227(4):279, 1979. 
[2] György Buzsáki. Rhythms of the Brain. Oxford University Press, 2006.

[3] György Buzsáki and Walter Freeman. Editorial overview: brain rhythms and dynamic coordination. Current opinion in neurobiology, 31:v, 2015.

[4] Marco Bocchio, Sadegh Nabavi, and Marco Capogna. Synaptic plasticity, engrams, and network oscillations in amygdala circuits for storage and retrieval of emotional memories. Neuron, 94(4):731-743, 2017.

[5] Maoz Shamir, Oded Ghitza, Steven Epstein, and Nancy Kopell. Representation of time-varying stimuli by a network exhibiting oscillations on a faster time scale. PLoS Comput Biol, 5(5):e1000370, 2009.

[6] Aryeh Hai Taub, Rita Perets, Eilat Kahana, and Rony Paz. Oscillations synchronize amygdala-to-prefrontal primate circuits during aversive learning. Neuron, 97(2):291-298, 2018.

[7] Charles M Gray, Peter König, Andreas K Engel, and Wolf Singer. Oscillatory responses in cat visual cortex exhibit inter-columnar synchronization which reflects global stimulus properties. Nature, 338(6213):334, 1989.

[8] Pascal Fries, John H Reynolds, Alan E Rorie, and Robert Desimone. Modulation of oscillatory neuronal synchronization by selective visual attention. Science, 291(5508):1560-1563, 2001.

[9] Narcisse P Bichot, Andrew F Rossi, and Robert Desimone. Parallel and serial neural mechanisms for visual search in macaque area v4. Science, 308(5721):529-534, 2005.

[10] Bijan Pesaran, John S Pezaris, Maneesh Sahani, Partha P Mitra, and Richard A Andersen. Temporal structure in neuronal activity during working memory in macaque parietal cortex. Nature neuroscience, 5(8):805, 2002.

[11] Ryan T Canolty, Erik Edwards, Sarang S Dalal, Maryam Soltani, Srikantan S Nagarajan, Heidi E Kirsch, Mitchel S Berger, Nicholas M Barbaro, and Robert T Knight. High gamma power is phase-locked to theta oscillations in human neocortex. science, 313(5793):16261628, 2006.

[12] Alexandra J Mably and Laura Lee Colgin. Gamma oscillations in cognitive disorders. Current opinion in neurobiology, 52:182-187, 2018.

[13] Wei Cao, Shen Lin, Qiang-qiang Xia, Yong-lan Du, Qian Yang, Meng-ying Zhang, Yi-qing Lu, Jing Xu, Shu-min Duan, Jun Xia, et al. Gamma oscillation dysfunction in mpfc leads to social deficits in neuroligin 3 r451c knockin mice. Neuron, 97(6):1253-1260, 2018.

[14] Supriya Ghosh, T Rao Laxmi, and Sumantra Chattarji. Functional connectivity from the amygdala to the hippocampus grows stronger after stress. Journal of Neuroscience, 33(17):7234-7244, 2013.

[15] Peter J Uhlhaas and Wolf Singer. Neural synchrony in brain disorders: relevance for cognitive dysfunctions and pathophysiology. neuron, 52(1):155-168, 2006.

[16] Pascal Fries, Danko Nikolić, and Wolf Singer. The gamma cycle. Trends in neurosciences, 30(7):309-316, 2007.

[17] Alon Amir, Drew B Headley, Seung-Chan Lee, Darrell Haufler, and Denis Pare. Vigilance-associated gamma oscillations coordinate the ensemble activity of basolateral amygdala neurons. Neuron, 97(3):656-669, 2018.

[18] Cristin G Welle and Diego Contreras. New light on gamma oscillations. Neuron, 93(2):247-249, 2017.
[19] Thilo Womelsdorf, Pascal Fries, Partha P Mitra, and Robert Desimone. Gamma-band synchronization in visual cortex predicts speed of change detection. Nature, 439(7077):733, 2006.

[20] Yotam Luz and Maoz Shamir. Oscillations via spiketiming dependent plasticity in a feed-forward model. PLoS computational biology, 12(4):e1004878, 2016.

[21] Alex Roxin, Nicolas Brunel, and David Hansel. Rate models with delays and the dynamics of large networks of spiking neurons. Progress of Theoretical Physics Supplement, 161:68-85, 2006.

[22] Demian Battaglia, Nicolas Brunel, and David Hansel. Temporal decorrelation of collective oscillations in neural networks with local inhibition and long-range excitation. Physical review letters, 99(23):238106, 2007.

[23] Sarit Soloduchin and Maoz Shamir. Rhythmogenesis evolves as a consequence of long-term plasticity of inhibitory synapses. Scientific reports, 8(1):13050, 2018.

[24] Demian Battaglia and David Hansel. Synchronous chaos and broad band gamma rhythm in a minimal multi-layer model of primary visual cortex. PLoS Comput Biol, 7(10):e1002176, 2011.

[25] Maoz Shamir. Theories of rhythmogenesis. Current opinion in neurobiology, 58:70-77, 2019.

[26] Hideyuki Câteau and Tomoki Fukai. A stochastic method to predict the consequence of arbitrary forms of spike-timing-dependent plasticity. Neural Computation, 15(3):597-620, 2003.

[27] Robert Gütig, Ranit Aharonov, Stefan Rotter, and Haim Sompolinsky. Learning input correlations through nonlinear temporally asymmetric hebbian plasticity. Journal of Neuroscience, 23(9):3697-3714, 2003.

[28] Abigail Morrison, Markus Diesmann, and Wulfram Gerstner. Phenomenological models of synaptic plasticity based on spike timing. Biological cybernetics, 98(6):459478, 2008.

[29] Jonathan Rubin, Daniel D Lee, and H Sompolinsky. Equilibrium properties of temporally asymmetric hebbian plasticity. Physical review letters, 86(2):364, 2001.

[30] Sen Song and Larry F Abbott. Cortical development and remapping through spike timing-dependent plasticity. Neuron, 32(2):339-350, 2001.

[31] Yotam Luz and Maoz Shamir. The effect of stdp temporal kernel structure on the learning dynamics of single excitatory and inhibitory synapses. PloS one, 9(7):e101109, 2014.

[32] Guo-qiang Bi and Mu-ming Poo. Synaptic modifications in cultured hippocampal neurons: dependence on spike timing, synaptic strength, and postsynaptic cell type. Journal of neuroscience, 18(24):10464-10472, 1998.

[33] Melanie A Woodin, Karunesh Ganguly, and Mu-ming Poo. Coincident pre-and postsynaptic activity modifies gabaergic synapses by postsynaptic changes in cl- transporter activity. Neuron, 39(5):807-820, 2003.

[34] Aparna Suvrathan, Hannah L Payne, and Jennifer L Raymond. Timing rules for synaptic plasticity matched to behavioral function. Neuron, 92(5):959-967, 2016.

[35] Julie S Haas, Thomas Nowotny, and Henry DI Abarbanel. Spike-timing-dependent plasticity of inhibitory synapses in the entorhinal cortex. Journal of neurophysiology, 2006.

[36] Curtis C Bell, Victor Z Han, Yoshiko Sugawara, and Kirsty Grant. Synaptic plasticity in a cerebellumlike structure depends on temporal order. Nature, 
387(6630):278, 1997.

[37] Makoto Nishiyama, Kyonsoo Hong, Katsuhiko Mikoshiba, Mu-Ming Poo, and Kunio Kato. Calcium stores regulate the polarity and input specificity of synaptic modification. Nature, 408(6812):584-588, 2000.

[38] Robert C Froemke, Mu-ming Poo, and Yang Dan. Spiketiming-dependent synaptic plasticity depends on dendritic location. Nature, 434(7030):221-225, 2005.

[39] Thanos Tzounopoulos, Maria E Rubio, John E Keen, and Laurence $\mathrm{O}$ Trussell. Coactivation of pre-and postsynaptic signaling mechanisms determines cell-specific spike-timing-dependent plasticity. Neuron, 54(2):291301, 2007.

[40] Donald O Hebb. The organization of behavior. na, 1961.

[41] Nimrod Sherf and Shamir Maoz. Multiplexing rhythmic information by spike timing dependent plasticity. PLOS Computational Biology, 16(6):e1008000, 2020.

[42] Hideyuki Câteau, Katsunori Kitano, and Tomoki Fukai. Interplay between a phase response curve and spiketiming-dependent plasticity leading to wireless clustering. Physical Review E, 77(5):051909, 2008.

[43] Wulfram Gerstner, Richard Kempter, J Leo Van Hemmen, and Hermann Wagner. A neuronal learning rule for sub-millisecond temporal coding. Nature, 383(6595):7678, 1996.

[44] Matthieu Gilson, Moritz Bürck, Anthony N Burkitt, and J Leo van Hemmen. Frequency selectivity emerging from spike-timing-dependent plasticity. Neural computation, 24(9):2251-2279, 2012.

[45] Jan Karbowski and G Bard Ermentrout. Synchrony arising from a balanced synaptic plasticity in a network of heterogeneous neural oscillators. Physical Review E, 65(3):031902, 2002.

[46] Robert R Kerr, Anthony N Burkitt, Doreen A Thomas, Matthieu Gilson, and David B Grayden. Delay selection by spike-timing-dependent plasticity in recurrent networks of spiking neurons receiving oscillatory inputs. PLoS Comput Biol, 9(2):e1002897, 2013.

[47] Shane Lee, Kamal Sen, and Nancy Kopell. Cortical gamma rhythms modulate nmdar-mediated spike timing dependent plasticity in a biophysical model. PLoS Comput Biol, 5(12):e1000602, 2009.

[48] Timothée Masquelier, Etienne Hugues, Gustavo Deco, and Simon J Thorpe. Oscillations, phase-of-firing coding, and spike timing-dependent plasticity: an efficient learning scheme. Journal of Neuroscience, 29(43):1348413493, 2009.

[49] Lyle E Muller, Romain Brette, and Boris Gutkin. Spiketiming dependent plasticity and feed-forward input oscillations produce precise and invariant spike phase-locking. Frontiers in Computational Neuroscience, 5:45, 2011.

[50] Jean-Pascal Pfister and Peter Tass. Stdp in oscillatory recurrent networks: theoretical conditions for desynchronization and applications to deep brain stimulation. Frontiers in computational neuroscience, 4:22, 2010.

[51] Emre Aksay, Itsaso Olasagasti, Brett D Mensh, Robert Baker, Mark S Goldman, and David W Tank. Functional dissection of circuitry in a neural integrator. Nature neu- roscience, 10(4):494-504, 2007.

[52] Joshua Kim, Michele Pignatelli, Sangyu Xu, Shigeyoshi Itohara, and Susumu Tonegawa. Antagonistic negative and positive neurons of the basolateral amygdala. Nature neuroscience, 19(12):1636, 2016.

[53] Minoru Koyama and Avinash Pujala. Mutual inhibition of lateral inhibition: a network motif for an elementary computation in the brain. Current Opinion in Neurobiology, 49:69-74, 2018.

[54] Dezhe Z Jin and H Sebastian Seung. Fast computation with spikes in a recurrent neural network. Physical Review E, 65(5):051922, 2002.

[55] Tomoki Fukai and Shigeru Tanaka. A simple neural network exhibiting selective activation of neuronal ensembles: from winner-take-all to winners-share-all. Neural computation, 9(1):77-97, 1997.

[56] Maoz Shamir. The scaling of winner-takes-all accuracy with population size. Neural computation, 18(11):27192729, 2006.

[57] John Hertz, Anders Krogh, Richard G Palmer, and Heinz Horner. Introduction to the theory of neural computation. PhT, 44(12):70, 1991.

[58] Maxim Bazhenov, Ramon Huerta, and Brian H Smith. A computational framework for understanding decision making through integration of basic learning rules. Journal of Neuroscience, 33(13):5686-5697, 2013.

[59] Christian K Machens, Ranulfo Romo, and Carlos D Brody. Flexible control of mutual inhibition: a neural model of two-interval discrimination. Science, 307(5712):1121-1124, 2005.

[60] Oran Zohar and Maoz Shamir. A readout mechanism for latency codes. Frontiers in computational neuroscience, 10:107, 2016.

[61] Simon M Danner, Natalia A Shevtsova, Alain Frigon, and Ilya A Rybak. Computational modeling of spinal circuits controlling limb coordination and gaits in quadrupeds. Elife, 6:e31050, 2017.

[62] Jessica Ausborn, Abigail C Snyder, Natalia A Shevtsova, Ilya A Rybak, and Jonathan E Rubin. State-dependent rhythmogenesis and frequency control in a half-center locomotor cpg. Journal of neurophysiology, 119(1):96-117, 2018.

[63] Guillaume Pernelle, Wilten Nicola, and Claudia Clopath. Gap junction plasticity as a mechanism to regulate network-wide oscillations. PLoS computational biology, 14(3):e1006025, 2018.

[64] Osame Kinouchi and Mauro Copelli. Optimal dynamical range of excitable networks at criticality. Nature physics, 2(5):348-351, 2006.

[65] Woodrow L Shew, Hongdian Yang, Shan Yu, Rajarshi Roy, and Dietmar Plenz. Information capacity and transmission are maximized in balanced cortical networks with neuronal avalanches. Journal of neuroscience, 31(1):5563, 2011.

[66] Oren Shriki and Dovi Yellin. Optimal information representation and criticality in an adaptive sensory recurrent neuronal network. PLoS computational biology, 12(2):e1004698, 2016. 